\title{
Ceratocone: fatores prognósticos
}

\author{
Keratoconus:prognosis factors
}

\author{
Rosana Molina Saraiva Elias ${ }^{1}$ \\ César Lipener ${ }^{2}$ \\ Ricardo Uras ${ }^{3}$ \\ Luis Pavês ${ }^{4}$
}

Trabalho realizado no Setor de Lente de Contato da Universidade Federal de São Paulo (UNIFESP).

${ }^{1}$ Médica colaboradora no Setor de Lente de Contato e Refração da Universidade Federal de São Paulo (UNIFESP). São Paulo (SP).

2 Médico chefe do Setor de Lente de Contato da UNIFESP. São Paulo (SP).

${ }^{3}$ Médico chefe do Setor de Refração da UNIFESP. São Paulo (SP).

${ }^{4}$ Médico colaborador do Setor de Lente de Contato e Refração da UNIFESP. São Paulo (SP).

Endereço para correspondência: Rosana Molina Saraiva Elias. Rua Camé, 532 - São Paulo (SP)

CEP 03121-020

E-mail: rosanelias@terra.com.br

Recebido para publicação em 05.11.2004

Versão revisada recebida em 01.04.2005

Aprovação em 15.04.2005

Nota Editorial: Após concluída a análise do artigo sob sigilo editorial e com a anuência dos Drs. Renato Giovedi Filho e Marcelo Carvalho da Cunha sobre divulgação de seus nomes como revisores, agradecemos sua participação neste processo.

\begin{tabular}{l} 
RESUMO \\
\hline Objetivo: Estudar pacientes portadores de ceratocone numa faixa etária \\
menor do que a usual e tentar detectar algum fator que possa influenciar \\
no prognóstico e na evolução da doença. Métodos: Foram analisados \\
pacientes menores de 15 anos, com diagnóstico de ceratocone. Todos \\
se submeteram a um questionário, exame ocular e ceratoscopia compu- \\
tadorizada. Estes pacientes foram divididos em 3 grupos e submetidos \\
a um teste com lente de contato: 1) um grupo em que foi possível a \\
adaptação de lente de contato; 2 ) um grupo em que foi indicado o \\
transplante penetrante de córnea; 3 ) um grupo em que foi indicada a \\
correção com óculos. Resultados: Pacientes com acuidade visual de \\
20/40 ou maior, geralmente não evoluíram para transplante no período \\
estudado. Ao contrário, pacientes com visão de $20 / 60$ ou menor, evoluí- \\
ram para transplante penetrante mais rapidamente. Ao serem estudados \\
casos em faixa etária menor(10-15 anos), 44\% evoluíram para transplante. \\
Observa-se que o ápice corneano tem localização muito variável, o que \\
explica os diferentes níveis de dificuldade na adaptação de lentes de \\
contato. Todos os pacientes com ápice deslocado superiormente e \\
valores maiores que 66 D foram encaminhados para cirurgia, ao passo que \\
olhos com valores menores que 66 D tiveram sucesso na adaptação. Nos \\
olhos com ápice central, a adaptação foi mais fácil. Nos casos de ápice \\
inferior não foi possível determinar um padrão para indicação de cirurgia. \\
Nos olhos adaptados com lente de contato e curvatura menor que 51,10 D \\
houve menos chance de evolução para transplante penetrante de córnea, \\
quando comparados com pacientes de curvatura 57,60 ou maior. Con- \\
clusão: Pacientes com curvaturas maiores que 57,40 acuidade menor que \\
20/60 e com idade menor de 18 anos tem uma chance maior de evoluir para \\
transplante de córnea. Quando o ápice do ceratocone é central a adaptação \\
de lente de contato fica mais fácil.
\end{tabular}

Descritores: Ceratocone/complicações; Prognóstico; Transplante de córnea; Lentes de contato; Acuidade visual

\section{INTRODUČ̃̃O}

O ceratocone é uma doença corneana degenerativa, não inflamatória, bilateral e assimétrica, progressiva, levando às inúmeras alterações na superfície da córnea. Caracteriza-se por afinamento central, protusão apical e astigmatismo irregular, com vários graus de cicatrização, ocasionando uma redução da acuidade visual. A córnea adquire forma cônica devido ao seu afinamento e protusão. Não existe infiltração celular ou vascularização. O cone pode ser redondo ou oval, podendo localizar-se próximo ao eixo visual, superior ou inferior a ele. Apesar de a etiologia permanecer indeterminada, existem várias associações incluindo doença atopeta, retardo men- 
tal, síndrome de Down, amaurose congênita de Leber, trauma ocular por lente de contato e doenças sistêmicas do colágeno (síndrome de Ehler-Danlos, síndrome de Marfan, osteogênese imperfeita).

A doença aparece tipicamente no final da adolescência e no adulto jovem, segundo um estudo realizado em 1994 com 2.723 pacientes, a idade média foi de 22,4 ano $^{(1)}$. Outro trabalho realizado em 1961 chegou à conclusão que a progressão do ceratocone ocorre entre 10 e 20 anos de idade ${ }^{(2)}$. Nem todos os casos de ceratocone evoluem da mesma forma. Aparentemente não existe nenhum agente que influencie a progressão do ceratocone. Vários estudos foram realizados para tentar predizer a progressão ou identificar fatores de risco para transplante. Foram evidenciadas em 1990 que córneas com curvaturas maiores que $50 \mathrm{D}$ e acuidade visual menor que 20/50 são fatores de alto risco para cirurgia, enquanto que sexo, idade e história de atopia são fatores não significativos para a evolução da doença $a^{(3)}$.

A introdução da ceratoscopia e fotoceratoscopia facilitou a compreensão da topografia da córnea normal e da anormal. A da videoceratoscopia computadorizada permitiu tanto a análise quantitativa quanto qualitativa de grandes áreas da superfície corneana. Alguns autores observaram pacientes com ceratocone e concluíram que $83 \%$ destes tinham o ápice deslocado inferiormente enquanto que apenas $17 \%$ tinham o ápice deslocado superiormente ${ }^{(4)}$.

O tratamento do ceratocone depende da severidade da doença. Os óculos são geralmente prescritos para os casos mais leves.

A correção com lente de contato é o tratamento mais freqüentemente utilizado para os pacientes com ceratocone, independente da fase, enquanto que o transplante penetrante de córnea está indicado nos casos em que não foi possível adaptar-se às lentes de contato ou em caso da existência de cicatriz corneana importante.

O objetivo deste trabalho é estudar pacientes numa faixa etária menor do que a usual e tentar detectar algum fator que possa influenciar no prognóstico e na evolução da doença.

\section{MÉTODOS}

Foram considerados neste trabalho todos os pacientes menores que 15 anos de idade, encaminhados para o Setor de Lente de Contato e Refração da UNIFESP, com diagnóstico de ceratocone, entre janeiro e dezembro de 1994. Os dados foram obtidos desde janeiro de 1994 através de questionário que constava de: idade, raça, profissão, história da doença atual e uso de lente de contato prévio. Foram verificados os antecedentes pessoais, especialmente os de alergia, tanto sistêmico como ocular (história de conjuntivite alérgica, rinite, asma, e/ou eczema) e os antecedentes familiares.

Os critérios diagnósticos para ceratocone incluíram a história clínica, exame biomicroscópico (estrias de Vogt, anel de Fleisher, afinamento central, cicatriz corneana, abaulamento de córnea, reflexo irregular na retinoscopia e topografia corneana computadorizada).

Em todos os pacientes envolvidos nesse estudo foi realizado exame oftalmológico que incluía: acuidade visual com correção (com óculos ou lente de contato), biomicroscopia, ceratometria, diâmetro corneano, diâmetro pupilar, fenda palpebral e topografia corneana com topógrafo Eye-sys CAS- $3^{\mathrm{A}}$. Para olhos em que as medidas ceratométricas eram maiores que $60 \mathrm{D}$, foi assumido um valor igual a $60 \mathrm{D}$.

Deste estudo foram excluídos olhos submetidos a transplante penetrante de córnea ou pacientes com menos de quatro meses de acompanhamento.

Todos os pacientes foram submetidos ao teste com lente de contato, inicialmente com lentes esféricas rígidas. Nas córneas em que não foi possível a adaptação, foram feitos testes com lentes bi curvas (Soper).

A seleção da lente de contato inicial foi feita a partir da curvatura mais plana das medidas ceratométricas e do diâmetro da córnea. Considerou-se como insucesso de adaptação da lente de contato, paciente com intolerância a ela, com visão menor que 20/50 e/ou incapacidade de adaptação.

Os pacientes em que não foi possível a adaptação das lentes de contato foram encaminhados para o Setor de Córnea para realização do transplante penetrante de córnea. Pacientes com ceratocone incipiente foi também tentada correção com óculos.

Após os testes de adaptação de lente de contato, os pacientes foram divididos em três grupos, conforme o resultado da adaptação: um grupo em que foi possível a adaptação de lente de contato; um grupo em que foi indicado o transplante penetrante de córnea e um grupo em que foi indicada a correção com óculos.

\section{RESULTADOS}

Foram estudados prospectivamente 30 olhos de 15 pacientes portadores de ceratocone entre janeiro/94 e dezembro/94, com idade entre 10 a 15 anos (média de12,8 anos). Destes, 8 eram do sexo feminino $(53,3 \%)$ e 7 do sexo masculino $(46,6 \%)$. 13 eram da raça branca $(86,6 \%)$ e 2 da raça negra $(13,33 \%)$. Dos 30 olhos, foram excluídos $3(10 \%)$ com transplante penetrante de córnea prévio à data da primeira consulta.

Em relação aos antecedentes pessoais, 8 pacientes $(53,3 \%)$ tinham alguma forma de atopia (rinite, asma e/ou conjuntivite alérgica). Nenhum dos pacientes apresentava eczema alérgico. Em relação aos antecedentes familiares, 3 referiam alguma forma de atopia entre parentes diretos (pai, tios e irmãos).

Dos 27 olhos, $4(13,33 \%)$ estavam em uso de lentes de contato e $2(6,66 \%)$ haviam usado lente de contato pelo menos uma vez durante o curso clínico da doença. Os outros pacientes negavam uso prévio de lente de contato antes da primeira consulta.

Nos 27 olhos examinados a ceratometria variou de 46,50 D no eixo mais plano (média de $56,40 \mathrm{D}$ ) a $60,00 \mathrm{D}$ no eixo mais curvo (média de 59,50 D). 
Dos 27 olhos em que foi tentada adaptação, em 14 (51\%) foi possível adaptar a lente de contato em: 1 (3\%) foi indicado uso de óculos e em 12 (44\%) foi indicado transplante penetrante de córnea. Os motivos que levaram a indicação do transplante foram: piora no quadro clínico com baixa da acuidade visual e intolerância ao uso de lente de contato ou adaptação inadequada. A indicação para transplante penetrante de córnea ocorreu em um período de 2 a 6 meses de evolução, sendo em todos os casos por piora da tolerância (Tabela 1).

Em um paciente ( 2 olhos) foi adaptada lente de contato gelatinosa correspondendo a $14,2 \%$ dos casos testados. No restante dos olhos (12 olhos), foram adaptadas lentes de contato rígidas gás permeáveis. A curva base das lentes de contato adaptadas variou de $45 \mathrm{D}$ a $69 \mathrm{D}$ (média de 54,8 D).

Nos pacientes em que foi indicado transplante penetrante de córnea por baixa de acuidade visual ou intolerância, a curva base média foi de $61,3 \mathrm{D}$ (portanto maior do que quando comparado com os pacientes em que foi possível a adaptação da lente de contato).

A acuidade visual variou de 20/400 a 20/20 com a melhor correção possível. Foi indicado transplante penetrante de córnea em pacientes com acuidade visual com lente de contato menor que 20/60, embora esse valor não seja um padrão rígido, já que alguns pacientes conseguiram usar lente de contato mesmo com essa visão.

Em relação à topografia computadorizada, foram examinadas 26. Em 6 pacientes não foi possível determinar-se a ceratometria através da topografia. Em 11 olhos o ápice era deslocado inferiormente, 12 superiormente e em 3 era central.

Em relação à presença de atopia, 15 olhos, correspondendo a 8 pacientes, tinham conjuntivite alérgica e/ou rinite associada ao ceratocone. Destes, em 8 olhos $(53 \%)$ foi indicado transplante penetrante de córnea e em $7(46,6 \%)$ foi adaptada lente de contato. Nestes pacientes a média ceratométrica foi de 55,25x58,60D.

Foram realizadas 15 topografias nos olhos dos pacientes com rinite e/ou conjuntivite, com média ceratométrica de $56,38 \times 61 \mathrm{D}$ (em dois, não foi possível avaliar as medidas). Os valores obtidos foram maiores que os obtidos por ceratômetro. O ápice do cone tinha média de 66,73 D (48 a 95 D). Destes 15 olhos, $6(40 \%)$ tinham o ápice do ceratocone desviado inferiormente, $7(46,66 \%)$ superiormente e $2(13,33 \%)$ eram centrais.

\begin{tabular}{|lc|}
\hline \multicolumn{2}{|c|}{ Tabela 1. Causas de indicação de transplante de córnea } \\
Causas & Número de pacientes \\
Intolerância & 1 \\
Baixa acuidade visual & 4 \\
Adaptação ruim & 2 \\
Baixa acuidade visual e intolerância & 3 \\
Baixa acuidade visual e adaptação ruim & 1 \\
Intolerância e adaptação ruim & 1 \\
Baixa acuidade visual e Intolerância & 2 \\
Total & 14 \\
\hline
\end{tabular}

Pacientes sem história de atopia tinham em média ceratometria de $57 \times 60$ D. Nesses pacientes foram realizadas 11 topografias, obtendo-se uma média da ceratometria de 55,2x60 D (semelhante à medida na ceratometria com ceratômetro), sendo que em seis pacientes não foi possível medir-se a curvatura. A média das medidas do ápice corneano foi de 72,27 D (53 a 95 D) sendo que 4 olhos tinham o ápice deslocado superiormente, 5 inferiormente e 2 centralmente.

Ao se relacionar a localização do ápice corneano através da topografia computadorizada e o sucesso de adaptação da lente de contato notou-se o seguinte: dos 10 olhos em que se conseguiu adaptar lente de contato, 4 tinham ápice deslocado inferiormente, 3 superiormente (valor do ápice menor que $67 \mathrm{D}$ ) e 3 centrais. Quatro olhos, apesar de se conseguir uma adaptação razoável, evoluíram para transplante; 3 tinham ápice deslocado inferiormente com valores entre 64 e $84 \mathrm{D}$ e 1 tinha ápice deslocado superiormente e medida de 86 D. Em relação aos 11 olhos em que se foi indicado transplante, 7 tinham um ápice deslocado superiormente com medida maior que $66 \mathrm{D}, 3$ eram inferiores com medidas entre 63 e 69 D e 1 era central.

\section{DISCUSS ÃO}

\section{I) AV $\mathbf{x}$ TX}

Em nosso trabalho, pacientes com acuidade visual de 20/40 ou maior, geralmente não evoluíram para transplante por essa indicação no período estudado. Ao contrário, pacientes com visão de 20/60 ou menor evoluíram para transplante penetrante mais rapidamente. Na literatura foi demonstrada uma mesma associação para pacientes com acuidade visual menor que $6 / 12^{(1)}$.

\section{II) CB x TX}

Da mesma forma, em nosso estudo verificamos que pacientes com lente de contato adaptadas e com curvatura menor que 51,1 D (8 olhos) têm uma chance menor de transplante, quando comparados a pacientes que adaptaram lente de contato com curva base maior que 57,6 D (4 olhos).

Alguns autores consideram que o raio da curva base da lente de contato ${ }^{(5)}$ seria o único parâmetro que prediz o transplante, outros consideram ${ }^{(2)}$ que a curvatura da córnea é um fator importante para se determinar os riscos de transplante e alguns caracterizam curvatura ${ }^{(3)}$ maior que $50 \mathrm{D}$ e baixa de visão menor que 20/50.

\section{III) IDADE $x$ TX}

Em nosso estudo verificamos que $44 \%$ dos casos foram indicados transplante.

Há relato de também não ter sido encontrado relação com a idade, sexo e história de atopia ${ }^{(3)}$. E evolução de $29 \%$ dos pacientes para transplante penetrante (lembrando que este estudo foi realizados com pacientes mais velhos).

Em nossa série, ao serem estudados casos em uma faixa 
etária menor (10-15 anos de idade), 44\% dos casos evoluíram para transplante.

Já em uma observação com pacientes jovens, menores que 18 anos de idade notou-se uma progressão mais rápida para cirurgia do que pacientes numa faixa etária maior ${ }^{(1)}$. O mesmo foi observado em nossa série.

\section{IV) TOPOGRAFIA $x$ TX}

Segundo pudemos observar, o ápice corneano tem uma localização muito variável e com isso existe uma maior ou menor dificuldade de adaptação de lente de contato. Em nossa série, 11 olhos $(42,6 \%)$ tinham o ápice corneano deslocado superiormente, $11(42,6 \%)$ inferiormente e 4 centrais $(15,3 \%)$. Todos os pacientes com ápice deslocado superiormente e valores maiores que $66 \mathrm{D}$ foram encaminhados para cirurgia enquanto que aqueles com valores menores que $66 \mathrm{D}$ tiveram sucesso na adaptação. Nos pacientes com ápice central, a adaptação foi mais fácil (em 3 olhos foi possível a adaptação). Quando o ápice é inferior não foi possível determinar-se um padrão para indicação de cirurgia. Um autor notou ${ }^{(4)}$ que em $83 \%$ dos olhos a porção mais curva da córnea localizava-se inferiormente e $17 \%$ superiormente, porém não há qualquer relação entre a topografia corneana e a dificuldade de adaptação da lente de contato.

\section{CONCLUSÃO}

Pacientes com curvaturas maiores que 57,40, acuidade menor que 20/60 e com idade menor de 18 anos têm uma chance maior de evoluir para transplante de córnea. Quando o ápice do ceratocone é central a adaptação de lente de contato fica mais fácil.

\section{ABSTRACT}

Purpose: To study keratoconus disease carrier patients in an age range under the usual one and try to detect any factor which can influence in disease evolution and prognosis. Methods: Patients under 15 years old with a keratoconus diagnosis where analyzed. All of them answered a questionnaire, and were submitted to ocular examination and also computerized ceratoscopy. These patients were divided into three groups and went through a contact lens test: 1) it was possible for this group to adapt to contact lenses; 2) a cornea transplant for this group was recommended; 3) for this group it was suggested that they use glasses. Results: Patients who had 20/40 or greater visual acuity usually did not progress to transplant in the studied period. On the other hand, patients who had 20/ 60 or less acuity, progress to a deep transplant very quickly. Other cases, related to a lower age range (10-15 years old) pregressed to a transplant. We can observe that the cornea apex has a very unsteady location, which explains the different difficulty levels regarding adaptation to contact lenses. In all patients who had a superior out of place apex and values greater than $66 \mathrm{D}$ adaptation was successful. In the central apex eyes the adaptation was easier. In the inferior apex eyes it was not possible to set a standard to indicate the person for surgery. In the eyes which were adapted to the contact lenses and whose curvature was smaller than $51.10 \mathrm{D}$ there were fewer chances of evolution to a cornea transplant, on comparison with patients who had a curvature of 57.60 or even greater. Conclusion: Patients who have curvatures greater than 57.40, acuity lower than 20/60 and are under 18 years old have more chances to evolve to a cornea transplant. When the ceratocone apex is central the contact lens adaptation becomes easier.

Keywords: Keratoconus/complications; Prognosis; Corneal transplantation; Contact lenses; Visual acuity

\section{REFERÊNCIAS}

1. Tuft SJ, Moodaley LC, Gregory WM, Davison CR, Buckey RJ. Prognostic factors for the progression of keratoconus. Ophthalmology. 1994;101(3):439-47.

2. Amsler M. [Some data on the problem of keratoconus]. Bull Soc Belge Ophtalmol. 1961;129:331-54. French

3. Lass JH, Lembach RG, Park SB, Hom DL, Fritz ME, Svilar GM, et al Clinical management of keratoconus. A multicenter analysis. Ophthalmology. 1990;97(4):433-45.

4. McMahon TT, Robin IB, Scarpulla KM, Putz JL. The spectrum of topography found in keratoconus. CLAO J. 1991;17(3):198-204

5. Woodward EG. Contact lenses in abnormal ocular conditions. Keratoconus. In: Phillips AJ, Stone I, editors. Contact lenses: a textbook for practitioner and student. $3^{\text {rd }}$ ed. London: Butterworth's; 1989. p.49.

6. Dana MR, Puts JL, Vinna MA, Sugar J, McMahon TT. Contact lens failure in keratoconus management. Ophthalmology. 1992;99(8):1187-92.

\section{Ao enviar um artigo para publicação, leia ATENTAMENTE as instruções para autores, constante no final de cada fascículo.}

